\title{
Determinantes microeconómicos de la pobreza multidimensional en la República Dominicana
}

\author{
Por Stephanie Reyes Pérez \& Joel Peguero Marte*
}

\begin{abstract}
Resumen
En este trabajo se presenta el análisis sobre la evolución y los determinantes microeconómicos de la pobreza multidimensional para la República Dominicana durante el periodo noviembre-abril 20072015, utilizando como insumo la Encuesta Nacional de la Fuerza de Trabajo provista por el Banco Central de la República Dominicana. Se abarca la pobreza desde el enfoque de las capacidades de Amartya Sen y el método de pobreza multidimensional propuesto por la Comisión Económica América Latina y el Caribe (CEPAL) y el Departamento de Desarrollo Sostenible y Pobreza de la Universidad de Oxford (OPHI), que engloba dimensiones más allá del ingreso, tales como: condiciones de la vivienda, servicios básicos, estándar de vida y educación, que a su vez se subdividen, para un total de 15 carencias, considerando pobres a las personas que posean un $25 \%$ de estas privaciones. El estudio fue sustentado en modelos econométricos, con el objetivo de determinar la incidencia de variables socioeconómicas en la probabilidad de ser pobre. Se implementaron modelos de corte transversal y Pseudo-Panel para observar la evolución de la pobreza bajo el amparo de la metodología de (Deaton, 1995), agrupando por cohortes a un grupo de individuos con características similares e invariables a través del tiempo.
\end{abstract}

Palabras clave: pobreza multidimensional; indicadores microeconómicos; movilidad social, República Dominicana.

Códigos del JEL: B21, C23, I32.

\footnotetext{
* Reyes: Escuela de Economía, Instituto Tecnológico de Santo Domingo (INTEC) y Analytica Inteligencia Económica y Estrategia. Dirección: Calle Boys Scouts \#16, Ensanche Naco, Distrito Nacional, República Dominicana. Tel.: 809683 5988. (email: stephreyesp@gmail.com). Peguero: Escuela de Economía, Instituto Tecnológico de Santo Domingo (INTEC) y Ayuntamiento del Distrito Nacional. Dirección: Avenida Jimenéz Moya, Distrito Nacional, República Dominicana. Tel.: 809535 1181. (email: joelpegueromarte@gmail. com). Agradecimientos: ambos autores agradecemos a Dios por cada logro, nuestros padres y el soporte brindado por los profesores del INTEC.
} 


\title{
Microeconomics determinants of multidimensional poverty in the Dominican Republic
}

\author{
By Stephanie Reyes Pérez \& Joel Peguero Marte*
}

\begin{abstract}
This paper presents the analysis of the evolution and microeconomic indicators of multidimensional poverty in the Dominican Republic (November-April 2007-2015), using the National Labor Force Survey provided by the Central Bank of Dominican Republic. Multidimensional poverty is approached from both Amartya Sen's capacities point of view and the method provided by the Economic Commission for Latin America and the Caribbean and the Department of Sustainable Development and Poverty of Oxford University. This is to include dimensions beyond income, such as tenement conditions, basic services, living standard and education. These subdivide into a total of 15 deficiencies, considering a person is poor when they have $25 \%$ of these deficiencies. The investigation was sustained with econometric models to understand the incidence the socioeconomic variables have in the probability of a person being poor. Both the cross-sectional and Pseudo-Panel methods were implemented to observe the evolution of poverty trough Deaton's method (1995), grouping by cohort individuals with similar characteristics that are invariable with time.
\end{abstract}

Keywords: multidimensional poverty; microeconomic indicators; social mobility; Dominican Republic.

JEL classification codes: B21, C23, I32.

\footnotetext{
* Reyes: Department of Economics, Instituto Tecnológico de Santo Domingo (INTEC) and Analytica Inteligencia Económica y Estrategia. Address: Street Boys Scouts \#16, Ens. Naco, Distrito Nacional, Dominican Republic. Tel.: 809683 5988. (email: stephreyesp@, gmail.com). Peguero: Department of Economics, Instituto Tecnológico de Santo Domingo (INTEC) and City Council of the National District. Address: Ave. Jimenéz Moya, Distrito Nacional, Dominican Republic. Tel.: 809535 1181. (email: joelpegueromarte@gmail.com). Acknowledgements: we thank God for every achievement, ours parents and the suport of our professors at INTEC.
} 


\section{Introducción}

\section{Planteamiento del problema}

La pobreza representa una privación del bienestar de las personas, por lo que superarla es uno de los objetivos del gobierno de la República Dominicana. Por tanto, medir correctamente la pobreza representa un reto, debido a la alta gama de formas en que esta puede limitar el bienestar de los individuos. Dentro de los beneficios de la medición correcta de la pobreza está que permite conocer las causas de esta y, de ese modo, mitigar sus efectos a partir de la implementación de políticas públicas. Los economistas enfocan sus estudios y análisis en dar soluciones a los problemas sociales, considerando la pobreza uno de los temas más destacados, desde finales del siglo XIX e inicios del siglo XX. El concepto y la medición de la pobreza provocó una apertura conceptual vertiginosa, donde los intelectuales de la época trataban, en primer lugar, de tener un concepto que abarcara los aspectos determinantes de la pobreza para, posteriormente, encontrar un método que de igual forma englobara la definición exacta de la misma.

\section{Propósito de la investigación}

Los objetivos de este trabajo de investigación son, en primer lugar, medir la pobreza a partir de agregados socioeconómicos, para evitar caer en el paradigma del ingreso y, de esta forma, observar cómo influyen estas variables sobre ella. Segundo, calcular la proporción de personas que vive en estado de pobreza en la República Dominicana. En adición, conocer la incidencia de aquellas variables de carácter social, tales como la salud, el último logro académico alcanzado, las variables relacionadas con quién es el jefe del hogar, el estado de la vivienda, el acceso al agua potable; así como la inclusión de otros elementos como la carencia de ciertos electrodomésticos y el hecho de poseer electricidad en el hogar. 


\section{Preguntas de investigación}

i. ¿Cuáles son las variables socioeconómicas significativas que influyen directamente en la determinación del concepto de pobreza?

ii. ¿Cuál es la diferencia de género existente en la probabilidad de ser pobres dependiendo de si el jefe del hogar es hombre o mujer?

iii. ¿Cuál ha sido la evolución de la pobreza multidimensional dentro del periodo 2007-2015?

\section{Marco teórico}

\section{Revisión de la literatura relacionada}

El término pobreza es complejo, pues se refiere a vivir una vida tolerable que supone la inclusión de aspectos como tener una vida larga y saludable, una buena educación, un buen servicio médico, además de poseer libertad política, respeto de los derechos humanos, seguridad personal, acceso a un trabajo productivo y bien remunerado y participación en la vida comunitaria. No obstante, dada la naturalidad de que algunas variables que constituyen la "calidad de vida" de las personas no pueden ser contabilizadas (como la libre asociación, la libertad de expresión, entre otras), el estudio de la pobreza se ha restringido a los aspectos cuantificables y materiales, relacionados con el "nivel de vida".

Según la Comisión Económica para América Latina (CEPAL), las mediciones de pobreza basadas exclusivamente en los ingresos monetarios proporcionan una evaluación incompleta del estándar de vida. La identificación de los pobres a partir de sus ingresos corrientes es una aproximación a la capacidad de consumo de los hogares por medio del mercado y no capta directamente el acceso a los bienes públi- 
cos (educación, salud, infraestructura básica) que no son adquiridos con el ingreso, lo que hace que la correlación entre ingreso y bienestar se desgaste (CEPAL, 2013).

Tal como expresa el Observatorio de la competitividad de la República Dominicana, la pobreza multidimensional busca ampliar la medición tradicional de la pobreza según los ingresos a otras dimensiones. En particular, esta analiza la relación entre las dimensiones de la pobreza: ¿hay relación de sustitución o complementariedad en materia de las carencias en ingresos, salud, educación y vivienda?, una persona que no es pobre en ingresos, ¿puede ser pobre en otras dimensiones? (Larrañaga, 2007).

Oxford Poverty and Human Development Initiative (OPHI), junto al Programa de Desarrollo de las Naciones Unidas (en inglés, United Nations Development Programme), creó el Índice de Pobreza Multidimensional, una medida internacional de pobreza aguda que cubre más de 100 países en desarrollo. Este complementa las bases de ingreso tradicional capturando las depravaciones severas que cada persona enfrenta al mismo tiempo con relación a la educación, la salud y los estándares de vida (Competitividad, s.f.).

La insaciable búsqueda de una definición concreta de pobreza, desde el siglo XX, ha generado una clasificación de esta según el enfoque de que se trate: Enfoque financiero o monetario, Enfoque económico, Enfoque de la desigualdad y Enfoque relativista.

En el 2005, el Banco Mundial reveló que existen al menos cuatro motivos para medir la pobreza. Primero, mantener a los pobres en la agenda, ya que si la pobreza no se midiera sería fácil olvidarse de ellos, debido a que el tema se podría ignorar si son estadísticamente invisibles. El segundo motivo es la capacidad de identificar la pobreza si se desea intervenir directamente con el propósito de reducirla o aliviarla. Tercero, para monitorear, evaluar y dar seguimiento a los programas y las políticas de intervención elaboradas para la población 
pobre. Y, por último, para evaluar la efectividad de las instituciones cuya principal meta es combatir la pobreza (The World Bank, 2005).

La dificultad generada por la medición de la pobreza bajo los supuestos del ingreso y las líneas de pobreza llevó a Amartya K., filósofo y economista bengalí, ganador del Premio Nobel de Economía en 1998, junto con el economista, político y diplomático tailandés Anand Panyarachun, a crear el IPH (Índice de Pobreza Humana), que mide privaciones en las dimensiones del desarrollo humano.

Según afirmaron Sen y Panyarachun en 1997, al medir la pobreza bajo variables microeconómicas se trata de abarcar los aspectos de vivir una vida tolerable, donde interactúan las tasas de esperanza de vida, alfabetización de los adultos y mortalidad de niños. Esto debido a que el ingreso no cubre todas las necesidades materiales. Dimensiones importantes del bienestar como la salud y la educación tienden a quedar fuera del rango de cobertura de los ingresos (Larrañaga, 2007).

De igual forma, Osvaldo Larrañaga, autor del informe Estudios estadísticos y prospectivos: la medición de la pobreza en dimensiones distintas al ingreso, plantea que la caracterización exclusiva de la población según sus ingresos provee una información errónea del bienestar de esa población. La investigación se limitará a utilizar aspectos económicos del bienestar que requieren acceso a bienes y servicios que tienen costo de oportunidad y conforman el ámbito tradicional de la pobreza.

En adición a los elementos necesarios para definir la pobreza de Sen, mencionados anteriormente (identificación y agregación), se agrega la comparación. Esta es propia de la medición multidimensional de la pobreza y se refiere a poder comparar el déficit de las distintas dimensiones del bienestar. Una de las formas de medir la pobreza multidimensional es tomar en cuenta el estado de salud de la persona. Existen dos tipos de indicadores relevantes, a) aquellos que miden la esperanza de vida o la esperanza de vida ajustada por discapacidad y 
b) los que miden las dimensiones de la salud, a partir de micro datos arrojados en encuesta de hogares o de personas. Para los fines de la investigación, se utilizará el indicador b.

La medición de la educación en el análisis de la pobreza multidimensional se realiza a través de indicadores de acceso o de logros. Cabe destacar que los indicadores relacionados con acceso están compuestos por la cobertura del sistema educacional y la tasa de deserción y de egresos de los distintos niveles educativos. Por otro lado, los logros se miden a través de los resultados en las pruebas de rendimiento escolar y del nivel de competencias alfabéticas en la población adulta. Es recomendable trabajar con indicadores relacionados con los logros, debido a que están estrechamente relacionados con el bienestar o el funcionamiento de las personas (Larrañaga, 2007).

Los autores Alkire y Foster de la Universidad de Oxford desarrollan una revisión a la medición de la pobreza multidimensional y crean una nueva metodología para medirla; en la actualidad, se utiliza su literatura como base para el cálculo de dicha pobreza. Esta metodología consiste en replantear los supuestos que propone Sen y, según los autores, radica en un método de identificación donde se extienden los enfoques tradicionales de intersección y unión, y una clase de mediciones de pobreza que satisface una variedad de propiedades deseables, incluyendo la descomponibilidad (Alkire \& Foster, 2007).

\section{Antecedentes de la medición de la pobreza en la República Dominicana}

La recuperación de la estabilidad macroeconómica del país en 1991 ha sido la base para que la República Dominicana experimente un notable y estable crecimiento económico. Pese a este crecimiento y a la reducción de la pobreza para la década de los noventa, donde el porcentaje de individuos por debajo de la línea de pobreza disminuyó de $37 \%$, en 1986 , a un $34 \%$, en 1992 , descendiendo drásticamente a un $28 \%$, en 1998; una proporción de la población no se benefició, 
principalmente las áreas rurales, tal como lo expresa el informe $R e$ pública Dominicana Informe sobre la pobreza: la pobreza en una economía de alto crecimiento (1986-2000), presentado por el Banco Mundial (2001).

Se evidenció una fuerte correlación entre la pobreza y los indicadores deficientes de salud. Como factor relevante, se destaca que un $20 \%$ de los niños en las regiones más pobres sufren de malnutrición. De igual forma, se observó la presencia de una correlación fuerte entre pobreza y educación, plasmando que, a mayor logro educacional del jefe del hogar, menos probabilidad tiene ese hogar de ser pobre. En cuanto a la ausencia de servicios básicos, tales como electricidad, agua y saneamiento, estos presentaron una fuerte correlación con la pobreza; sin embargo, las transferencias domésticas y las remesas del exterior poseen una fuerte incidencia en la reducción de la pobreza (Banco Mundial, 2001).

La Oficina Nacional de Estadísticas (ONE) es la institución encargada oficialmente de dirigir las encuestas y de dar monitoreo a gran parte de los indicadores económicos. El informe citado previamente, expuesto por el Banco Mundial, exhibe que de igual forma el Banco Central y las instituciones privadas, al no contar con una recolección sistemática por parte de la ONU, se sumergen de forma más activa en la medición de bienestar en el país.

Específicamente, el Banco Central implementó la Encuesta Nacional de Gastos e Ingresos de los Hogares (ENGIH), siendo esta la más completa para 1998. Dentro de las instituciones privadas que se vieron inmersas en la medición de bienestar en el país, se destacaron en 1986: Tuffs University, con la Encuesta Nacional de Ingreso-Consumo, la Fundación Economía y Desarrollo, que condujo la Encuesta de Ingresos y Gastos, financiados por la USAID.

Tomando como base las informaciones de Antonio Morillo, analista de pobreza dominicano, se presenta en el gráfico 1 la diversidad de 
cifras de pobreza monetaria existentes para la República Dominicana hasta finales de la década de los noventa (Morillo, 2008).

Gráfico 1: Divergencias en las eStimaCiOnES DE POBREZA MONETARIA EN LA RePÚBLICA DOMINICANA, PERIODO 1990-1999

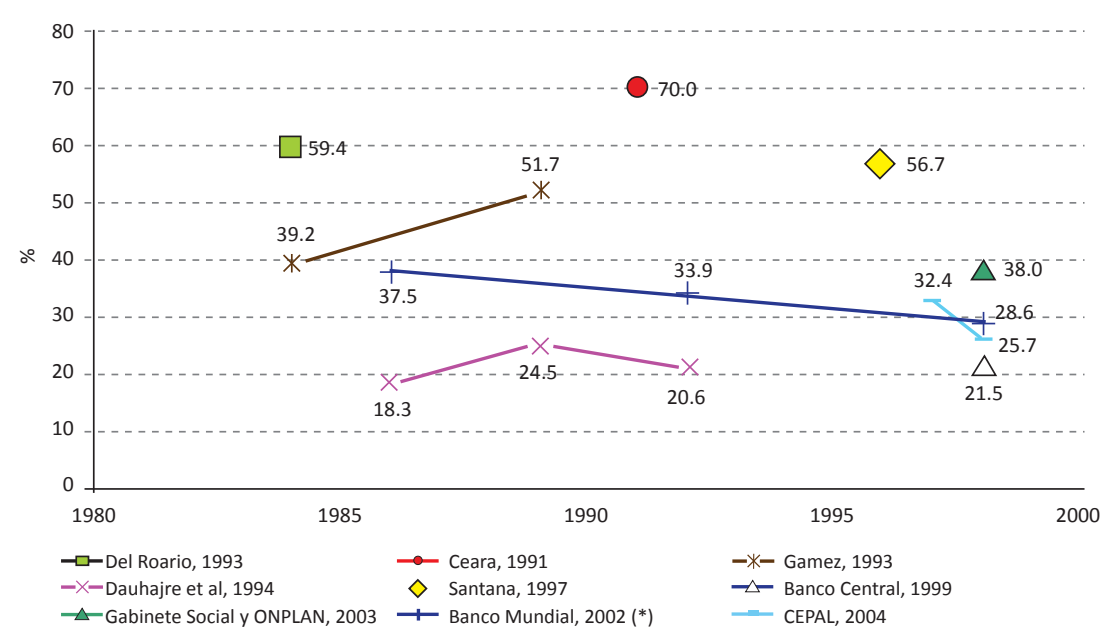

Fuente: Informe Medición de la pobreza monetaria mediante la EFT, 2000-2008².

El crecimiento económico experimentado en la década de los noventa pierde auge por la aguda crisis financiera y económica acontecida en el periodo 2003-2004. Los niveles de pobreza y los ingresos de los pobres experimentaron una escasa mejoría durante la bonanza de crecimiento registrada entre 1997-2000, traduciéndose en el aumento de un $50 \%$ en la tasa de pobreza y la duplicación del número de pobres extremos del país; en cifras, el $15.6 \%$ de la población pasó a ser pobre, es decir, con ingresos insuficientes para cubrir el costo mínimo de sus necesidades básicas, y un 4.2\%, alrededor de 670 mil personas, cayó en la pobreza extrema (Banco Mundial y Banco Interamericano de Desarrollo, 2006).

\footnotetext{
${ }^{1}$ Medición de la pobreza monetaria mediante las Encuestas de Fuerza de Trabajo (EFT) del Banco Central de la República Dominicana: propuesta metodológica y resultados 20002008 (Morillo, 2008).
} 
Antonio Morillo expresa que, tras los problemas presentados por la medición de la pobreza monetaria, diversos autores buscaron alternativas y otras metodologías para medir la pobreza multidimensional en la República Dominicana. A partir de 1997, se elabora por primera vez en el país el mapa de la pobreza, sustentado en datos del VII Censo Nacional de Población y Viviendas, en 1993. Poseía un atlas con 72 mapas nacionales y provinciales, donde se desplegaban dos indicadores: la tasa de pobreza general y la extrema (Morillo, 2014).

A partir de 1997, el Banco Central implementa la Encuesta Nacional de la Fuerza de Trabajo (ENFT), considerada por los autores como la única que permite comparaciones consistentes en el tiempo.

Gracias a la carencia de las metodologías y las fuentes de datos oficiales de amplia aceptación para el monitoreo de la pobreza, se generaron estimaciones de pobreza que muestran un amplio rango y tendencias inconsistentes. El Banco Mundial y el BID, junto con el programa $\mathrm{MECOVI}^{2}$, facilitan en 2006 un proceso inter-agencial, incluyendo la formación de un comité técnico oficial interagencial coordinado por el BCRD, para examinar distintas metodologías para la medición de la pobreza.

Para el año 2012, la República Dominicana aún carecía de una metodología oficial consensuada para la medición de la pobreza monetaria. En ese año, el proyecto inter-institucional mencionado anteriormente se encontraba en la fase de culminación. En este escenario las estimaciones realizadas de pobreza monetaria estaban basadas en el ámbito de organismos de cooperación de índole internacional, con las Encuestas de la Fuerza de Trabajo (EFT) del Banco Central y la CEPAL, que elabora estimaciones para el país con una metodología que incorpora líneas de pobreza nacionales (Morillo, 2012).

\footnotetext{
${ }^{2}$ Encuesta para la medición de Condiciones de Vida en América Latina.
} 
Gráfico 2: Divergencias en las estimaciones de pobreZA MONETARIa en la República Dominicana, PERIOdo 2000-2006

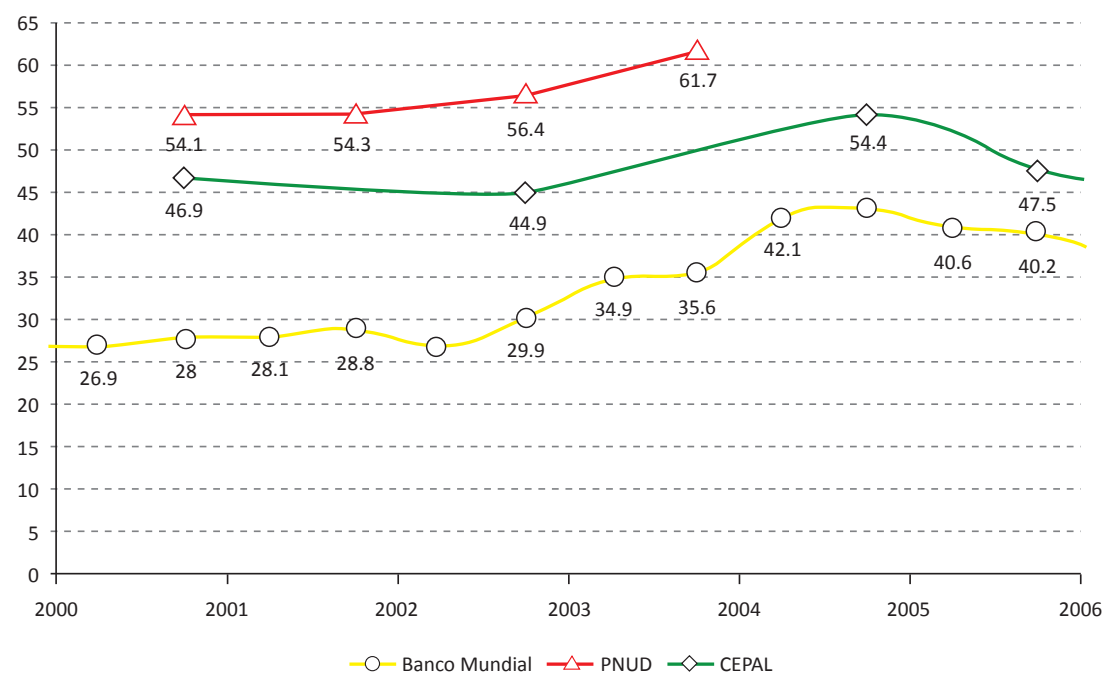

Fuente: Informe Medición de la pobreza monetaria mediante la EFT, 2000-2008³.

\section{Diagnóstico}

La medición de la pobreza en la República Dominicana en las últimas tres décadas se ha basado en las estimaciones de la pobreza monetaria. Pero, es a partir del año 2012, con el desarrollo y la adopción consensuada de una metodología oficial para la medición de la pobreza monetaria que se superan los problemas relacionados con la calidad de los datos de ingresos familiares utilizados para mediciones y líneas de pobreza (Morillo, 2014).

Las mediciones se obtienen a partir de los datos sobre los ingresos recogidos en la Encuesta Nacional de la Fuerza de Trabajo (ENFT) y son levantadas semestralmente por el Banco Central de la República Dominicana. En la actualidad existen 34 bases levantadas en los

\footnotetext{
${ }^{3}$ Medición de la pobreza monetaria mediante las Encuestas de Fuerza de Trabajo (EFT) del Banco Central de la República Dominicana: Propuesta metodológica y resultados 20002008 (Morillo, 2008).
} 
meses de abril y octubre, desde el año 2000 al 2016. El MEPyD define la pobreza monetaria como la situación en la que prevalece un déficit respecto al monto de los recursos, representado por los ingresos considerados necesarios para que una persona pueda adquirir una canasta de alimentos mínimos para su consumo y otras necesidades (que no son alimentos) consideradas esenciales; es decir, vestidos, calzados, vivienda, cuidados del hogar, salud, educación, transporte, entre otras.

La metodología oficial implementada para la medición de pobreza en República Dominicana se determina sobre la base de tres insumos: a) El indicador de bienestar, definido a partir del ingreso disponible en los hogares, construido con la Encuesta Nacional de Fuerza de Trabajo. b) La Canasta Básica Alimentaria (CBA); y c) La Canasta Básica no Alimentaria (CBNA).

Pese a que las mediciones de pobreza en la República Dominicana están basadas en el enfoque monetario, en el mapa de pobreza de la República Dominicana de 2014 se utiliza el Método ICV como medida principal para la medición de la pobreza desde el punto de vista multidimensional, utilizando como insumo los datos provistos por la Encuesta Nacional de la Fuerza de Trabajo (ENFT). De igual forma, se implementa de forma complementaria una versión ampliada del método $\mathrm{NBI}^{4}$, que involucra quince carencias sociales.

Según el autor, estos indicadores se emplean desde hace dos décadas, pero no son reconocidos como métodos oficiales de la medición de pobreza multidimensional. La razón se debe a algunos problemas

\footnotetext{
${ }^{4}$ Método de las Necesidades Básicas Insatisfechas (Método NBI), donde se identifican hogares que sufren de carencia o privación de bienes y servicios, recursos u oportunidades que posibilitarían su subsistencia y desarrollo. Consiste en identificar hogares que sufren este tipo de privaciones. Las privaciones utilizadas convencionalmente en el país son: i) vivir en una vivienda con piso de tierra; ii) no tener agua corriente por tubería conectada a la red pública; iii) no poseer servicios sanitarios; iv) no disponer de energía eléctrica; v) vivir en hacinamiento (más de 3.5 personas por dormitorio); vi) el hogar tiene al menos un miembro adulto en edad laboral que es semi-analfabeto (Morillo, 2014 y 2015).
} 
del método NBI, como las subjetividades en la selección de las variables y las carencias, además de que no se utilizan métodos estadísticos ni en la selección de variables, ni en los criterios para definir la carencia, ni en las ponderaciones de lugar.

Abarcando los Objetivos del Milenio para la creación del IPM$\mathrm{RD}$, donde el primer objetivo de la lista es erradicar la pobreza extrema y el hambre para la revisión de 2010, la meta planteada en 1990 era reducirla a la mitad, entre 1990 y 2015, por debajo de la línea nacional de extrema pobreza. La proporción de la población en pobreza extrema en 1992 se encontraba en $10.8 \%$ y en 2009 era de $10.4 \%$; del mismo modo, el coeficiente de brecha de pobreza pasó de 3.3 a 2.9 en 2009 y el ingreso nacional correspondiente al quintil más pobre de la población pasa de 4.3 a $3.8 \%$, siendo todos estos indicadores signos claros y concisos de que ese objetivo no se cumpliría para 2015 (Ministerio de Economía Planificación y Desarrollo, 2010).

Es a partir de 2010 que se crea la Estrategia Nacional de Desarro1lo, con el objetivo de instituir un futuro de creciente bienestar. Dentro de los objetivos de alta prioridad, según el eje estratégico, se encuentran: implantar un sistema educativo de calidad que capacite para el aprendizaje continuo a lo largo de la vida y propicie el desarrollo humano; universalizar el aseguramiento en salud para garantizar el acceso a servicios de esa índole; disminuir y aliviar la pobreza mediante un efectivo y eficiente sistema de protección social y desarrollo de capacidades para generar ingresos (Ministerio de Economía Planificación y Desarrollo, 2010).

Según el SUIBEN, los objetivos de contar con el Índice de Pobreza Multidimensional radican en: i) contar con un indicador capaz de dar a conocer otros factores determinantes de la pobreza no considerados en la métrica actual por el SUIBEN; ii) obtener una medición de pobreza que incluya aspectos relacionados con el bienestar psicológico, la seguridad física y medioambiental; y iii) asegurar comparabilidad 
con otros países que utilizan el IPM a nivel regional (SUIBEN \& Vicepresidencia de la República Dominicana, 2016).

Las dimensiones e indicadores que pretenden abordar el IPM-RD corresponden a los sectores: salud, educación, brecha social, vivienda, vulnerabilidad medioambiental y bienestar humano. Los aspectos que toma en cuenta el sector salud son: mortalidad en la niñez, aseguramiento en salud, alimentación, acceso a servicios de salud o sanitarios y cuidado infantil. Desde el punto de vista de educación, abarca el alfabetismo, el rezago y la deserción; por su parte, la brecha social incorpora el acceso a las TICs, la seguridad ciudadana y el acceso a los espacios recreativos, culturales y deportivos.

A finales de junio de 2017, el Ministerio de Economía, Planificación y Desarrollo emite el primer informe preliminar sobre el Índice de Pobreza Multidimensional para América Latina (IPM-LA): una aplicación para República Dominicana 2005-2016. El informe obedece al propósito fundamental de complementar el análisis de la pobreza monetaria en la República Dominicana con una medición multidimensional, que permite cuantificar la pobreza más allá del ingreso y de las privaciones que la determinan, en pos de dar soporte al diseño de políticas públicas orientadas al mejoramiento de la calidad de vida de la población dominicana.

Las estimaciones arrojaron que la tasa de incidencia ajustada de pobreza multidimensional en la República Dominicana disminuyó 11.8 puntos porcentuales en los últimos cuatro años, reduciéndose de $34.9 \%$ (en 2012) a $23.1 \%$ (en 2016); destacando que la misma permaneció ligeramente estancada en los años previos. Se utilizaron como insumo los datos provistos por la Encuesta Nacional de Fuerza de Trabajo publicadas por el Banco Central para el periodo 2005-2016 (Morillo, El índice de Pobreza Multidimensional Para América Latina (IMP-LA): Una aplicación para la República Dominicana 2005-2016 (Informe Preeliminar), 2017). 


\section{Metodología}

Para responder las interrogantes antes planteadas y, de igual forma, satisfacer los objetivos y propósitos previamente presentados se parte, en primer lugar, de un análisis teórico deductivo, es decir, analizando teóricamente el concepto de pobreza multidimensional y luego se hace un recorrido por la historia de la forma en que esta es medida. Luego, a partir de modelos econométricos, pasar a determinar o medir la pobreza en la República Dominicana partiendo de determinantes microeconómicos para observar cómo esta fue evolucionando en el periodo estudiado.

En un primer modelo econométrico, a partir de los determinantes socioeconómicos, se generará una variable proxy para pobreza que estará compuesta por condiciones del hogar, tales como poseer pared exterior de palma o de desecho, no tener pared interior en el hogar, carencia de cocina e inodoro en la vivienda, nivel de educación, carencia de necesidades básicas, entre otras variables. Las variables explicativas del modelo provienen del modelo teórico en el que se basará la investigación, el paper escrito por Luis Quinn titulado Determinantes de pobreza y vulnerabilidad social en República Dominicana para el periodo 2010-2012, donde se emplea un modelo de probabilidad Probit para medir la pobreza bajo determinantes microeconómicos y macroeconómicos. Cabe destacar que a pesar de que el modelo se basa en la investigación de Quinn, fueron añadidas y sustraídas otras variables explicativas consideradas de gran significancia para la pobreza, siguiendo la metodología de corte transversal.

En un segundo modelo econométrico, esta investigación recurrirá a una metodología de datos de pseudo-panel. La razón por la cual se trabajará con dicha metodología se debe a que la Encuesta Nacional no toma, año tras año, a los mismos individuos. Se aplicará un modelo de efecto aleatorio Probit para calcular la probabilidad de que un individuo sea pobre en la República Dominicana, además de ver su trayectoria a través de los años del análisis. 
La metodología de pseudo-panel nace en 1993 a través del profesor escocés, merecedor del Premio Nobel de Economía en 2015, Angus Stewart Deaton. Esta metodología permite dar un seguimiento artificial de los individuos. A pesar de que esta herramienta ha sido escasamente explotada, es útil para el análisis que se busca realizar en este trabajo de investigación.

En primer lugar, para construir una base de datos de pseudo-panel es necesario realizar cohortes, definidos como grupos de individuos que poseen características similares que no varían a través del tiempo. Posteriormente, se calculan los valores medios de las variables a analizar para cada cohorte. La finalidad de las cohortes es crear grupos homogéneos entre sí y heterogéneos entre cohortes (Sahagún, 2016).

Con el fin último de mitigar o corregir los errores de medición, diferentes autores en la práctica han justificado que el problema de error de medición puede ser ignorado siempre que el número de observaciones por cohorte sea al menos de 100 observaciones (Sahagún, 2016).

\section{Data}

La data implementada como insumo para el desarrollo de este modelo es la Encuesta Nacional de Fuerza de Trabajo, desde 2007 hasta 2015, en el periodo noviembre-abril, realizada por el Banco Central de la República Dominicana; la base de datos está compuesta por ocho estratificaciones: vivienda, hogar, miembro, ocupación, ingresos del exterior, remesas calculadas y tasas moneda, las cuales están compuestas por otras variables.

La ENFT utilizada para el desarrollo del modelo es un pseudo-panel para el semestre noviembre-abril de 2007-2015; las muestras fueron recogidas de forma aleatoria simple, proporcionando un total de 261,089 observaciones. 
Tal como se muestra en la tabla 1, en este trabajo de investigación se están analizando 73,083 hogares y 269,081 personas para el periodo de estudio.

TABLA 1: TAMAÑo DE MUESTRA EFeCTIVA DE HOGARES Y PERSONAS DE LA ENCUESTA NACIONAL DE TRABAJO ENFT, PERIODO 2007-2015

\begin{tabular}{ccc}
\hline Encuesta & Hogares & Personas \\
\hline Abril 2007 & 7,569 & 28,045 \\
Abril 2008 & 8,351 & 30,760 \\
Abril 2009 & 8,362 & 30,709 \\
Abril 2010 & 8,317 & 30,493 \\
Abril 2011 & 8,258 & 29,380 \\
Abril 2012 & 8,155 & 28,711 \\
Abril 2013 & 8,215 & 28,491 \\
Abril 2014 & 8,005 & 27,772 \\
Abril 2015 & 7,851 & 26,728 \\
Total & 73,083 & 269,081 \\
\hline
\end{tabular}

Fuente: elaboración propia con los datos proporcionados por la ENFT, abril 2007-2015.

Luego de tener lista la base de datos, se crea la variable proxy para pobreza, la cual está compuesta por las variables que se muestran en la tabla debajo, tomadas como referencia de los indicadores y criterios de privación en la aplicación de la metodología IMP-LA para la República Dominicana. Luego de crear esta variable proxy, como se implementará un modelo de probabilidad Probit y según la metodología utilizada por el Ministerio de Economía Planificación y Desarrollo, se consideró pobre a las personas que poseían al menos el $25 \%$ de las carencias o privaciones que se presentarán a continuación: 
TABla 2: INDiCADORES DE PRIVACIONES O CARENCIAS UTILIZADAS BASADAS EN LA METODOLOGÍA IPM-LA

\begin{tabular}{|c|c|c|}
\hline Dimensión & Indicador & Definición de la privación \\
\hline \multirow{3}{*}{ Vivienda } & $\begin{array}{l}\text { Precariedad de } \\
\text { los materiales de } \\
\text { construcción de la } \\
\text { vivienda }\end{array}$ & $\begin{array}{l}\text { Viviendas con piso de tierra, } \\
\text { techo (materiales de desecho, } \\
\text { yagua, zinc), pared interior } \\
\text { (yagua, zinc, sin pared interior, } \\
\text { cartón y palma), pared exterior } \\
\text { (yagua, zinc, materiales de } \\
\text { desecho, cartón, palma) }\end{array}$ \\
\hline & Hacinamiento & $\begin{array}{l}\text { Viviendas con } 3 \text { o menos } \\
\text { dormitorios y } 9 \text { o más personas } \\
\text { viviendo en la misma }\end{array}$ \\
\hline & $\begin{array}{l}\text { Tenencia insegura de la } \\
\text { vivienda }\end{array}$ & $\begin{array}{l}\text { Habitan en viviendas donadas } \\
\text { por familiares o por el } \\
\text { Gobierno, barrancones o } \\
\text { prestadas }\end{array}$ \\
\hline \multirow{3}{*}{ Servicios básicos } & Carencia de agua & No posee agua por red pública \\
\hline & Carencia de saneamiento & $\begin{array}{l}\text { Hogares que poseen letrinas o } \\
\text { no poseen servicios sanitarios }\end{array}$ \\
\hline & Carencia de energía & $\begin{array}{l}\text { Hogares que no poseen } \\
\text { alumbrado público }\end{array}$ \\
\hline \multirow[t]{2}{*}{ Estándar de vida } & $\begin{array}{l}\text { Carencia de bienes } \\
\text { duraderos }\end{array}$ & $\begin{array}{l}\text { Ausencia de nevera, lavadora y } \\
\text { abanico eléctrico }\end{array}$ \\
\hline & Inasistencia a la escuela & $\begin{array}{l}\text { Niños entre } 6 \text { y } 17 \text { años sin } \\
\text { educación }\end{array}$ \\
\hline Educación & $\begin{array}{l}\text { Logro educativo } \\
\text { insuficiente }\end{array}$ & $\begin{array}{l}\text { Hogares donde personas entre } \\
20 \text { y } 59 \text { años solo alcanzaron } \\
\text { completar la primaria y hogares } \\
\text { con personas mayores a } 60 \\
\text { años con educación primaria } \\
\text { completa }\end{array}$ \\
\hline
\end{tabular}

Fuente: elaboración propia basados en los criterios de privación y carencia para el cálculo del IPM-LA.

Para poder crear la variable proxy de pobreza, se sumaron todos los indicadores dicotómicos mostrados en la tabla anterior; se considerarán personas pobres aquellas que posean al menos 4 privaciones, es decir, que tengan carencias de servicios básicos, saneamiento, educación, estándar de vida y hogares precarios. 
Como resultado de lo mencionado anteriormente, se obtuvo que la tasa de incidencia ${ }^{5}$ para el periodo analizado muestra que la crisis financiera de 2008 tuvo repercusiones sobre las privaciones y carencias de las personas en República Dominicana. Las privaciones para el año 2007 se encontraban, aproximadamente, en un $42 \%$ y en 2008 aumentó a 53 puntos porcentuales; es a partir de 2012 donde el porcentaje de personas con privaciones empieza a tomar un sendero de reducción paulatino.

Gráfica 3: TASA DE INCIDENCIA 2007-2015

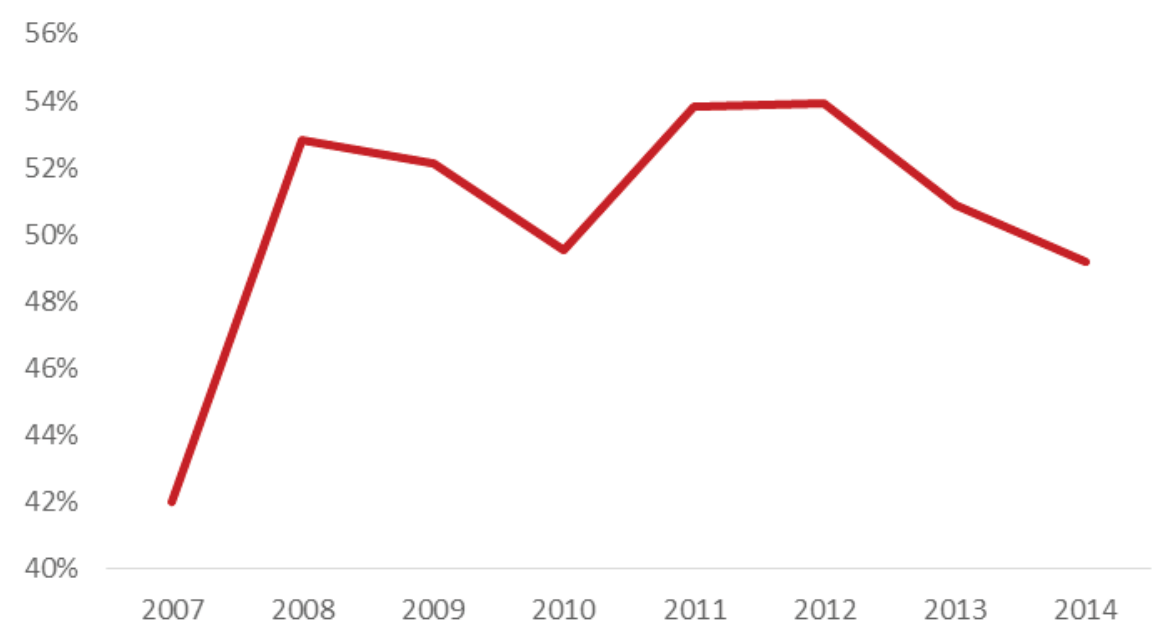

Fuente: elaboración propia con los datos proporcionados por la ENFT, abril 2007-2015.

Para el análisis de los efectos de las variables microeconómicas sobre la probabilidad de que una persona sea pobre (que posea al menos cuatro privaciones o carencias) se partirá del modelo de probabilidad no lineal Probit de corte transversal, para el periodo 2007-2015, destacando que los coeficientes de este modelo solo nos expresan la dirección del efecto; para saber los efectos de las variables independientes sobre la variable dependiente es necesario calcular los efectos marginales de las variables explicativas del modelo.

\footnotetext{
${ }^{5}$ Tasa de incidencia(H): Porcentaje de individuos que son pobres multidimensionales. $\mathrm{H}=\frac{q}{n} * 100$ [Morillo, El índice de Pobreza Multidimensional Para América Latina (IMP-LA): Una aplicación para la República Dominicana 2005-2016 (Informe Preeliminar), 2017]
} 
Para calcular la evolución de la variable pobreza, definida por los individuos que poseen al menos cuatro privaciones, el tratamiento idóneo para este tema sería la metodología de datos de panel, pero dado que no se siguen a las mismas personas, es necesario crear grupos con individuos que posean características invariables a través del tiempo, denominados cohortes. Las cohortes implementadas para el análisis de esta investigación son: fecha de nacimiento (compuesta por grupos de edades de 15 años), último nivel educativo alcanzado y sexo. Luego de poseer las cohortes definidas, se procede a limpiarlas. Según Deaton, las cohortes son representativas cuando poseen al menos 100 individuos por cohorte.

Luego de poseer las cohortes representativas, se procede a la realización del modelo de probabilidad Probit, tomando como variable dependiente la variable Pobreza y como variables explicativas las variables microeconómicas significativas sobre las privaciones y carencias de la persona (pobreza).

\section{Resultados de la investigación}

\section{Modelo de probabilidad Probit de corte transversal}

Debido a que se ha implementado un modelo de probabilidad Probit, los coeficientes poseen una interpretación totalmente distinta a la usual. Los coeficientes de la regresión de este tipo de modelo expresan la dirección del efecto sobre la probabilidad de éxito o fracaso de la variable dependiente. Para obtener el efecto preciso es necesario recurrir al cálculo de los efectos marginales (Medina, 2003).

Las variables microeconómicas implementadas con el objetivo de conocer su incidencia sobre la probabilidad de que una persona sea pobre son:

- Pobreza: variable dependiente del modelo; la misma es una variable dicotómica que toma valor cero $(0)$ cuando la persona no 
es considerada pobre. Valor determinado a partir de la metodología expuesta por Alkire y Foster (2007), todas las personas que posean al menos 4 carencias son consideradas pobres y toman el valor uno (1).

- Formal: variable dicotómica que expresa si el individuo labora en el sector económico formal. Toma valor uno (1) cuando el individuo forma parte de este sector y cero (0) cuando pertenece al sector informal.

- Ocupado: variable dicotómica definida por personas mayores de 10 años que estén trabajando y devengando un salario. Si el valor de esta variable es uno (1), se considera que la persona se encuentra ocupada; por el contrario, si la variable toma valor cero (0), entonces la persona se encuentra desocupada.

- Edad: es la única variable discreta asumida en el modelo. La misma expresa la edad de los individuos al momento de realizar la encuesta.

- Casado: variable binaria que expresa si el estado civil de la persona es casado u otro. Cuando la variable es igual a uno (1), se concluye que la persona se encuentra casada, mientras que si toma valor (0) el estado civil de la persona en estudio es diferente a casado, entiéndase, soltero, viudo, separado o divorciado.

- Posee televisor: variable dicotómica que representa la existencia de al menos un televisor en el hogar; si la misma acoge valor cero (0), refleja la carencia de televisor en el hogar y si acoge valor uno (1) indica que sí existe televisor.

- Hombre jefe del hogar: variable dicotómica que refleja si el jefe del hogar es hombre. Al tomar valor uno (1) implica que el jefe del hogar es el hombre y al tomar valor cero (0) refleja que la mujer es la jefa del hogar. 
- Calles asfaltadas: variable dicotómica que refleja si el sector donde reside la persona posee calles asfaltadas. Si dicha variable acoge valor cero ( 0 ), muestra que las personas no poseen calles asfaltadas; si acoge valor uno (1), expresa que sí las posee.

Por ende, el modelo de probabilidad no lineal Probit analizado con los signos esperados es el siguiente:

Pobreza $=\beta_{0}-\beta_{1}$ Ocupado $-\beta_{2}$ Formal $+\beta_{3}$ Edad $-\beta_{4}$ Casado $-\beta_{5}$ PoseeTv $+\beta_{6}$ HombreJefedeHogar $-\beta_{7}$ CallesAsfaltadas $-\beta_{8} \mathrm{Edad}^{2}+\mathrm{e}$.

Se espera que ante la presencia del hombre como jefe de hogar la probabilidad de ser pobre aumente. La variable Edad posee el signo positivo $(+)$ porque se espera que el efecto de la misma sea positivo para los primeros años del individuo, dado que depende de otras personas. A partir de los 18 años de edad hasta los 60 años, aproximadamente, se espera que el efecto sea negativo, por tal motivo se incluyó la variable $E d a d^{2}$ y, a partir de esto, se puede determinar el punto de inflexión, que muestra a partir de qué edad la probabilidad de ser pobre aumenta o disminuye.

Los resultados encontrados para los modelos de probabilidad no lineal Probit de corte transversal para el periodo analizado, destacando que se corrigió la heterocedasticidad aplicando errores robustos, revelaron las siguientes estimaciones:

Pobreza $=\beta_{0}-\beta_{1}$ Ocupado $-\beta_{2}$ Formal $+\beta_{3}$ Edad $-\beta_{4}$ Casado $-\beta_{5}$ PoseeTv $+\beta_{6}$ HombreJefedeHogar $-\beta_{7}$ CallesAsfaltadas $-\beta_{8} \mathrm{Edad}^{2}+\mathrm{e}$. 
Tabla 3: Resultados Modelo de probabilidad no lineal 2007-2015

\begin{tabular}{|c|c|c|c|c|c|}
\hline & (1) & (2) & (3) & (4) & (5) \\
\hline Variables & Pobreza 2007 & Pobreza 2008 & Pobreza 2009 & Pobreza 2010 & Pobreza 2011 \\
\hline \multirow[t]{2}{*}{ Ocupado } & $0.137 * * *$ & $0.0848 * * *$ & $0.161 * * *$ & $0.136 * * *$ & $0.164 * * *$ \\
\hline & $(0.0287)$ & $(0.0307)$ & $(0.0310)$ & $(0.0295)$ & $(0.0332)$ \\
\hline \multirow[t]{2}{*}{ Formal } & $-0.418 * * *$ & $-0.377 * * *$ & $-0.488 * * *$ & $-0.444 * * *$ & $-0.440 * * *$ \\
\hline & $(0.0284)$ & $(0.0313)$ & $(0.0313)$ & $(0.0297)$ & $(0.0331)$ \\
\hline \multirow[t]{2}{*}{ Edad } & $0.0313 * * *$ & $0.0212 * * *$ & $0.0214 * * *$ & $0.0241 * * *$ & $0.0235 * * *$ \\
\hline & $(0.00269)$ & $(0.00280)$ & $(0.00277)$ & $(0.00263)$ & $(0.00301)$ \\
\hline \multirow[t]{2}{*}{ Edadsqrt } & $-0.000313 * * *$ & $-0.000187 * * *$ & $-0.000219 * * *$ & $-0.000228 * * *$ & $-0.000231 * * *$ \\
\hline & $(3.05 \mathrm{e}-05)$ & $(3.13 \mathrm{e}-05)$ & $(3.09 \mathrm{e}-05)$ & $(2.94 \mathrm{e}-05)$ & $(3.36 \mathrm{e}-05)$ \\
\hline \multirow[t]{2}{*}{ Paga alquiler } & $-0.217 * * *$ & $-0.269 * * *$ & $-0.353 * * *$ & $-0.338 * * *$ & $-0.440 * * *$ \\
\hline & $(0.0249)$ & $(0.0271)$ & $(0.0267)$ & $(0.0259)$ & $(0.0287)$ \\
\hline \multirow[t]{2}{*}{ Casado } & $-0.560 * * *$ & $-0.525 * * *$ & $-0.634 * * *$ & $-0.611 * * *$ & $-0.589 * * *$ \\
\hline & $(0.0300)$ & $(0.0314)$ & $(0.0312)$ & $(0.0309)$ & $(0.0343)$ \\
\hline \multirow[t]{2}{*}{ Posee TV } & $-1.160 * * *$ & $-1.250 * * *$ & $-1.890 * * *$ & $-1.339 * * *$ & $-1.754 * * *$ \\
\hline & $(0.0702)$ & $(0.0784)$ & $(0.146)$ & $(0.0948)$ & $(0.0983)$ \\
\hline \multirow{2}{*}{$\begin{array}{l}\text { Calles } \\
\text { asfaltadas }\end{array}$} & $-0.894 * * *$ & $-0.821 * * *$ & $-0.863 * * *$ & $-0.785 * * *$ & $-0.806^{* * *}$ \\
\hline & $(0.0254)$ & $(0.0258)$ & $(0.0261)$ & $(0.0260)$ & $(0.0294)$ \\
\hline \multirow{2}{*}{$\begin{array}{l}\text { Hombre jefe } \\
\text { del hogar }\end{array}$} & $0.183 * * *$ & $0.131 * * *$ & $0.206 * * *$ & $0.174 * * *$ & $0.126 * * *$ \\
\hline & $(0.0316)$ & $(0.0331)$ & $(0.0333)$ & $(0.0321)$ & $(0.0356)$ \\
\hline \multirow[t]{2}{*}{ Constante } & $2.275 * * *$ & $2.625 * * *$ & $3.345 * * *$ & $2.653 * * *$ & $3.182 * * *$ \\
\hline & $(0.0811)$ & $(0.0890)$ & $(0.152)$ & $(0.101)$ & $(0.115)$ \\
\hline Observaciones & 22,556 & 24,602 & 24,792 & 24,684 & 23,899 \\
\hline
\end{tabular}




\begin{tabular}{|c|c|c|c|c|}
\hline & (6) & (7) & (8) & (9) \\
\hline Variables & Pobreza 2012 & Pobreza 2013 & Pobreza 2014 & $\begin{array}{c}\text { Pobreza } \\
2015\end{array}$ \\
\hline \multirow[t]{2}{*}{ Ocupado } & $0.119 * * *$ & $0.0943 * * *$ & $0.170 * * *$ & $0.145 * * *$ \\
\hline & $(0.0327)$ & $(0.0311)$ & $(0.0311)$ & $(0.0314)$ \\
\hline \multirow[t]{2}{*}{ Formal } & $-0.408 * * *$ & $-0.391 * * *$ & $-0.398 * * *$ & $-0.483 * * *$ \\
\hline & $(0.0329)$ & $(0.0312)$ & $(0.0313)$ & $(0.0303)$ \\
\hline \multirow[t]{2}{*}{ Edad } & $0.0261 * * *$ & $0.0226 * * *$ & $0.0176 * * *$ & $0.0191 * * *$ \\
\hline & (0.00299) & $(0.00277)$ & $(0.00283)$ & $(0.00277)$ \\
\hline \multirow[t]{2}{*}{ edadsqrt } & $-0.000277 * * *$ & $-0.000236 * * *$ & $-0.000179 * * *$ & $-0.000191 * * *$ \\
\hline & $(3.31 \mathrm{e}-05)$ & $(3.03 e-05)$ & $(3.09 \mathrm{e}-05)$ & $(3.04 \mathrm{e}-05)$ \\
\hline \multirow[t]{2}{*}{ Paga alquiler } & $-0.333 * * *$ & $-0.392 * * *$ & $-0.398 * * *$ & $-0.424 * * *$ \\
\hline & $(0.0285)$ & $(0.0273)$ & $(0.0273)$ & $(0.0263)$ \\
\hline \multirow[t]{2}{*}{ Casado } & $-0.581 * * *$ & $-0.613 * * *$ & $-0.582 * * *$ & $-0.587 * * *$ \\
\hline & $(0.0344)$ & $(0.0325)$ & $(0.0327)$ & $(0.0319)$ \\
\hline \multirow[t]{2}{*}{ Posee TV } & $-1.746 * * *$ & $-1.756 * * *$ & $-1.686^{* * *}$ & $-1.187 * * *$ \\
\hline & $(0.0923)$ & $(0.0885)$ & $(0.0894)$ & $(0.0850)$ \\
\hline \multirow{2}{*}{$\begin{array}{l}\text { Calles } \\
\text { asfaltadas }\end{array}$} & $-0.938 * * *$ & $-0.805^{* * *}$ & $-0.790 * * *$ & $-0.909 * * *$ \\
\hline & $(0.0320)$ & $(0.0302)$ & $(0.0304)$ & $(0.0302)$ \\
\hline \multirow{2}{*}{$\begin{array}{l}\text { Hombre jefe } \\
\text { del hogar }\end{array}$} & $0.189 * * *$ & $0.211 * * *$ & $0.187 * * *$ & $0.171 * * *$ \\
\hline & $(0.0354)$ & $(0.0337)$ & $(0.0338)$ & $(0.0323)$ \\
\hline \multirow[t]{2}{*}{ Constante } & $3.219 * * *$ & $3.169 * * *$ & $3.163 * * *$ & $2.745 * * *$ \\
\hline & $(0.105)$ & $(0.104)$ & $(0.103)$ & $(0.0957)$ \\
\hline Observaciones & 23,429 & 23,435 & 22,849 & 22,099 \\
\hline
\end{tabular}

Robust standard errors in parentheses

$* * * \mathrm{p}<0.01, * * \mathrm{p}<0.05, * \mathrm{p}<0.1$

Fuente: elaboración propia con los datos proporcionados por la ENFT, abril 2007-2015.

A partir de la tabla 3, se observa como en cada corte transversal o cada año posterior, a pesar de que son muestras diferentes, la dirección del efecto de las variables explicativas del modelo no presenta variaciones. Se observa que los efectos de todas las variables explica- 
tivas del modelo se comportan de acuerdo a lo esperado, exceptuando a la variable Ocupado. Si una persona está económicamente ocupada, según la estimación del modelo, posee un signo positivo, traduciéndose a que estar económicamente ocupado aumenta la probabilidad de ser pobre, efecto que no concuerda con la realidad. Este efecto contrario no tiene sentido desde la óptica económica y puede deberse al sesgo de la información, debido a que se observó en los datos de la ENFT que incluso niños de 9 años se encuentran económicamente ocupados. De igual forma, este sesgo puede responder a que no se están controlando otros factores exógenos y, por ende, se arrojen estos resultados, o a la inclusión de variables irrelevantes. No obstante, el modelo econométrico resultó, según pruebas estadísticas (Ruido blanco, Heterocedasticidad), un modelo adecuado para el análisis (Zarraga, Esteban, Orbe, \& Regúlez, 2009).

Vale la pena resaltar que todas las variables implementadas en el modelo son estadísticamente significativas al $1 \%$ del nivel de confianza. Tal como se expresó anteriormente, estos coeficientes solo muestran la dirección del efecto de las variables independientes sobre la probabilidad de éxito o fracaso de la variable dependiente. Para evaluar el efecto concreto se analizan los efectos marginales presentados a continuación:

Los efectos marginales miden el cambio en la probabilidad de la variable explicada del modelo, en este caso, Pobreza. Tal como se había mencionado, los efectos estimados de las variables explicativas son iguales a los efectos esperados, con excepción de la variable $\mathrm{Ocu}$ pado. Para el año 2007, se evidencia que, si una persona se encuentra económicamente ocupada, aumenta la probabilidad de ser pobre en $2.6 \%$, manteniendo todo lo demás constante. Resultado contradictorio, debido a que se espera que, si una persona se encuentra ocupada económicamente, devengue beneficios monetarios de esa actividad. 
Tabla 4: Efectos marginales de modelos de probabilidad Probit 2007-2015

\begin{tabular}{cccccc}
\hline & \multicolumn{5}{c}{ Efectos marginales } \\
Pobreza & 2007 & 2008 & 2009 & 2010 & 2011 \\
\hline Ocupado* & $\mathrm{dy} / \mathrm{dx}$ & $\mathrm{dy} / \mathrm{dx}$ & $\mathrm{dy} / \mathrm{dx}$ & $\mathrm{dy} / \mathrm{dx}$ & $\mathrm{dy} / \mathrm{dx}$ \\
Formal* & 0.02552 & 0.10605 & 0.17936 & 0.01956 & 0.01431 \\
Edad & -0.08879 & -0.56437 & -0.06974 & -0.07756 & -0.04867 \\
Edadsqrt & 0.00584 & 0.00266 & 0.00241 & 0.00349 & 0.00206 \\
Paga alquiler* & -0.00006 & -0.00002 & -0.00002 & -0.00003 & -0.00002 \\
Casado* & -0.04346 & -0.03828 & -0.04709 & -0.0564 & -0.04855 \\
Posee TV* & -0.12974 & -0.08616 & -0.10043 & -0.11844 & -0.08553 \\
Calles asfaltadas* & -0.1259 & -0.91756 & -0.09628 & -0.10418 & -0.09401 \\
Hombre jefe de & -0.16281 & -0.11174 & -0.10365 & -0.11219 & -0.07238 \\
hogar* & 0.0321 & 0.01564 & 0.0213 & 0.02361 & 0.01045 \\
\hline
\end{tabular}

\begin{tabular}{ccccc}
\hline & \multicolumn{3}{c}{ Efectos marginales } & \\
Pobreza & 2012 & 2013 & 2014 & 2015 \\
\hline & $\mathrm{dy} / \mathrm{dx}$ & $\mathrm{dy} / \mathrm{dx}$ & $\mathrm{dy} / \mathrm{dx}$ & $\mathrm{dy} / \mathrm{dx}$ \\
Ocupado* & 0.01049 & 0.01028 & 0.01988 & 0.0227 \\
Formal* & -0.0448 & -0.05162 & -0.05603 & -0.09101 \\
Edad & 0.00231 & 0.00247 & 0.00206 & 0.00299 \\
Edadsqrt & -0.00002 & -0.00003 & -0.00002 & -0.00003 \\
Paga alquiler* & -0.0349 & -0.05142 & -0.05561 & -0.07785 \\
Casado* & -0.07366 & -0.09451 & -0.09362 & -0.1207 \\
Posee TV* & -0.09684 & -0.11454 & -0.11145 & -0.10271 \\
Calles asfaltadas* & -0.08161 & -0.08155 & -0.08484 & -0.13101 \\
Hombre jefe de & 0.01535 & 0.02117 & 0.02039 & 0.02521 \\
hogar* & & & & \\
\hline
\end{tabular}

(*) Variable dicotómica

Fuente: elaboración propia con los datos proporcionados por la ENFT, abril 2007-2015.

Según las estimaciones, en el año 2009 el efecto marginal de la variable Ocupado mostró su valor más alto; si una persona se encontraba económicamente ocupada, se traducía en un aumento de 18 puntos porcentuales en la probabilidad de ser pobre. Si el hombre es el jefe del hogar, según los estimado, posee 3.2 puntos porcentuales de posibilidad de ser pobre con respecto a cuando la mujer es jefa del 
hogar, siendo en 2007 donde esta diferencia presenta su valor máximo con relación a los años analizados. Para el año 2015 se obtuvo que, si el hombre es jefe del hogar, posee $2.5 \%$ de probabilidad de poseer al menos el $25 \%$ de las privaciones respecto a cuando la mujer es la jefa del hogar, ceteris paribus ${ }^{6}$

La interpretación de los efectos marginales a partir de este punto serán el efecto de la variable microeconómica sobre la probabilidad de ser pobre, es decir, de que posea al menos el $25 \%$ de las privaciones para 2015. Si el individuo pertenece al sector formal, reduce la probabilidad de ser pobre en 9.1 puntos porcentuales; si la persona paga alquiler, reduce la probabilidad de ser pobre en $7.8 \%$; si el individuo posee calles asfaltadas, la probabilidad de poseer al menos el $25 \%$ de las carencias o privaciones se reduce en $8.2 \%$, con todo lo demás constante; resultado congruente con los presentados en el paper base (Quinn, 2013).

\section{Evolución de la pobreza pseudo-panel}

\section{Modelo de probabilidad Probit para pseudo-panel}

A diferencia de los datos de panel, los pseudo-paneles trabajan con cohortes, las cuales están compuestas por personas que poseen características comunes invariantes a lo largo del tiempo. Por ende, las observaciones de cada cohorte expresan la media de las observaciones de los individuos que conforman dicha cohorte. Es por tal razón que, en vez de trabajar con individuos como se haría en datos de panel, se trabaja con pseudo-individuos.

Debido a la variación de unidades a pseudo-individuos y a las observaciones presentadas en función de los promedios de los individuos de la cohorte, esto conlleva a una interpretación de los

\footnotetext{
${ }^{6}$ Supuesto económico que exhibe el efecto de una variable económica sobre otra, manteniendo sin variación las demás variables que puedan impactarle.
} 
coeficientes totalmente diferente. Es de vital importancia destacar que, al utilizar un modelo Probit binomial, todas las variables que forman parte del modelo deben ser dicotómicas. A continuación, se definen las cohortes utilizadas y las variables que conforman el modelo:

Cohortes o pseudo-individuos: las cohortes fueron realizadas a partir de grupos de individuos que hayan nacido en lapsos de 15 años, sexo y último logro educacional alcanzado, resultando 79 cohortes, de las cuales solo 49 eran válidas para el análisis, es decir, que poseían más de 100 observaciones por cohortes, según lo enunciado por Deaton (Sahagún, 2016).

- Pobreza: corresponde al promedio de personas consideradas pobres dentro de la cohorte; en adición, es una variable dicotómica que toma valor cero ( 0 ) cuando la persona no es considerada pobre.

- Ocupado: expresa el promedio de las personas que se encuentran ocupadas dentro de las cohortes. Está definida por personas mayores de 10 años que estén trabajando y devengando un salario. Si el valor de esta variable es mayor a 0.50 , se considera que la persona se encuentra ocupada y viceversa.

- Mujer jefa del hogar: representa al promedio de hogares dentro de la cohorte donde el jefe del hogar es mujer. Esta variable es dicotómica, por ende, si esta toma valor mayor a 0.5 se considera que la mujer es la jefa del hogar.

- Soltero: corresponde al promedio de las personas de la cohorte que se encuentran solteras. Si la variable toma valor 0 , entonces el promedio de las personas que están dentro de la cohorte poseen un estado civil distinto al de soltero.

- Posee TV: corresponde a la media de los individuos que poseen un televisor dentro de la cohorte. Si el valor que toma es mayor 
a 0.5 , se traduce que el promedio de las personas de esta cohorte posee un televisor en su hogar.

Después de realizar las manipulaciones necesarias para adecuar la data e implementar la metodología de pseudo-paneles, se realizan las estimaciones pertinentes al modelo de probabilidad Probit. El modelo que se pretende analizar es el siguiente:

Pobreza $=\beta_{0}-\beta_{1}$ Ocupado $-\beta_{2}$ Soltero $-\beta_{3}$ PoseeTv + $\beta_{4}$ Mujerjefedelhogar $+\mathrm{e}$

Se espera que, si una persona se encuentra actualmente trabajando y devengando un ingreso o beneficio, es decir, ocupada, esto se traduzca en una reducción de la probabilidad de ser pobre o de tener carencias o limitaciones dentro del hogar. Del mismo modo, se estima que, si una persona es soltera, se extrapole a una reducción sobre la probabilidad de ser pobre, dado que incurre en menos gastos para su manutención, en comparación con los demás estados civiles. Asimismo, ante la presencia de individuos con televisores en sus hogares, se estima una reducción en la probabilidad de ser pobres.

Al momento de analizar el efecto de la mujer como jefa de hogar, cabe resaltar que, debido a la segmentación existente por género del mercado laboral, específicamente en América Latina, y al compromiso doméstico que cargan sobre sus hombros las madres solteras, la obtención de un ingreso monetario resulta (en promedio) inferior al que puede obtenerse cuando el hombre es el jefe de hogar. El hecho de que estas mujeres deben hacerse responsables del trabajo doméstico y del sostén económico las induce a gastar una proporción de sus ingresos en la adquisición de bienes y servicios en el mercado, de forma que dediquen menos tiempo a este trabajo no remunerado (Geldstein, 1997). Por ende, se espera que el efecto de una mujer jefa de hogar sobre la probabilidad de ser pobre sea positivo. 
Previo a la estimación del modelo, debe observarse la correlación entre la variable dependiente e independiente del modelo. Para poder seguir adelante en el modelo se propone que las variables no posean una correlación mayor a 0.5 . Solamente la variable dicotómica Poseer TV en el hogar presenta una alta correlación con la variable dependiente Pobreza; luego todas las demás variables poseen una correlación menor a 0.5 , por lo que se procede a la estimación del modelo de probabilidad no lineal Probit.

A diferencia de los datos de panel, los pseudos-paneles trabajan con cohortes, que están compuestas por personas que poseen características comunes invariantes a lo largo del tiempo. Por ende, las observaciones de cada cohorte expresan la media de las observaciones de los individuos que la conforman. Es por tal razón que, en vez de trabajar con individuos como se haría en datos de panel, se trabaja con pseudo-individuos (Sahagún, 2016).

El modelo de probabilidad Probit para pseudo-panel fue estimado a través del método de efectos aleatorios, obteniendo los siguientes resultados:

$$
\begin{gathered}
\text { Pobreza }=11.81-2.25 \text { ocupado }+8.97 \text { soltero }-9.55 \text { poseetv } \\
-0.84 \text { mujerjefedelhogar }+e
\end{gathered}
$$

Las variables implementadas en el modelo son estadísticamente significativas a un $1 \%$ y $5 \%$ nivel de significancia, a diferencia de la variable Mujer jefa del hogar, que resulta ser estadísticamente no significativa y se deja en el modelo debido a que es lo referido por el modelo base. 
Tabla 5: Regresión Modelo Probit

\begin{tabular}{lc}
\hline Variables & $\begin{array}{c}\text { Modelo Probit } \\
\text { pobreza }\end{array}$ \\
\hline Ocupado & $-2.254^{* *}$ \\
& $(1.048)$ \\
Soltero & $8.973^{* *}$ \\
& $(3.961)$ \\
Posee televisión & $-9.550^{* * *}$ \\
Mujer jefa del hogar & $(3.201)$ \\
& -0.847 \\
Constante & $(1.355)$ \\
& $11.82 * * *$ \\
Observaciones & $(3.210)$ \\
Number of coh & 421 \\
\hline
\end{tabular}

Standard errors in parentheses $* * * \mathrm{p}<0.01, * * \mathrm{p}<0.05, * \mathrm{p}<0.1$

Fuente: elaboración propia a partir de los datos obtenidos de la ENFT 2007-2015, en el software estadístico Stata.

Según las estimaciones realizadas para el cálculo de la evolución de la pobreza, se obtuvo que, si las personas económicamente ocupadas crecen $1 \%$ dentro de una cohorte, estas poseen una probabilidad un $2.25 \%$ menor de ser pobres. Asimismo, si los pseudo-individuos solteros crecen un $1 \%$ dentro de la cohorte, tienen una probabilidad $8.97 \%$ mayor de poseer al menos el $25 \%$ de las carencias propuestas al inicio de la investigación. Las cohortes donde la mujer jefa del hogar crezca un $1 \%$ poseen $0.85 \%$ menos probabilidad de ser pobres que las cohortes donde el hombre como jefe del hogar crezca un $1 \%$.

Cabe destacar que, a diferencia del modelo de corte transversal, en este método econométrico si una persona se encuentra ocupada económicamente, disminuye la probabilidad de ser pobre, lo que evidencia el sesgo de la data sin estratificación alguna (cohortes) y de 
utilizar modelos de corte transversal para ver la evolución o la trayectoria de una variable a través del tiempo.

Se procedió a realizar un gráfico para percibir visualmente la evolución de la probabilidad de la variable definida Pobreza y, en adición, la tasa de incidencia, que mide la proporción de personas con carencias en el tiempo. Se obtuvo, tal como se observa en el gráfico a continuación, que ambas poseen un comportamiento idéntico.

GrÁFICO 4: TRAYECTORIA DEL PORCENTAJE DE PRIVACIONES Y LA TASA DE INCIDENCIA

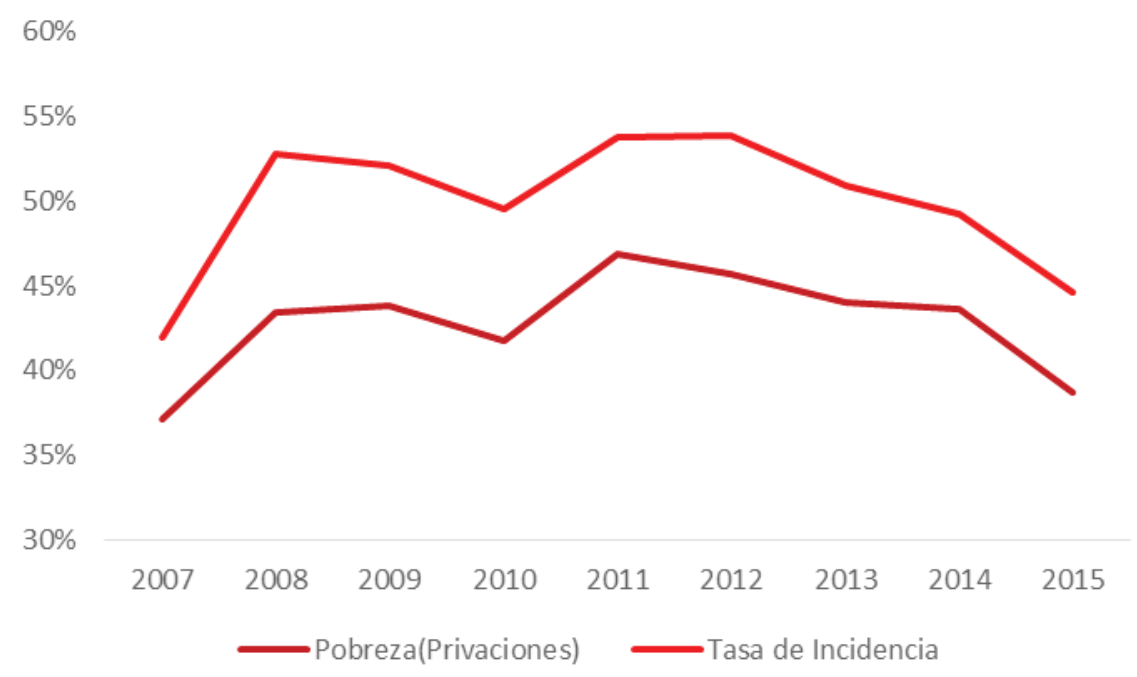

Fuente: elaboración propia a partir de los datos obtenidos de la ENFT 2007-2015, en Stata.

Se observa que la variable llamada Pobreza, que representa los hogares que poseen al menos el $25 \%$ de las privaciones asumidas y presentadas en el presente trabajo de investigación, posee el mismo comportamiento que la tasa de incidencia que refleja el porcentaje de personas pobres multidimensionales. Se observa un alza ligera de estas variables para el año 2008, por lo que se podría inferir que la crisis financiera de ese año tuvo repercusiones sobre dichas variables. Asimismo, se evidencia una caída pronunciada en el año 2010 en ambas variables. 
La única diferencia que presentan estas variables es que la tasa de incidencia presenta una tendencia paulatina a la baja a partir de 2012 y las variables que miden los hogares que poseen al menos el $25 \%$ de las privaciones presenta una disminución a partir de 2011, resultados que concuerdan con el Informe preliminar del Índice de Pobreza Multidimensional para República Dominicana, emitido por el Ministerio de Economía y Desarrollo.

\section{Conclusiones}

Luego de modelar los años desde 2007 hasta 2015, a través de un método de corte transversal y con un modelo econométrico Probit, utilizando el software estadístico Stata, se obtuvo que a través de los años las variables microeconómicas con fuerte significancia sobre la probabilidad de que un individuo sea pobre o no fueron varias, destacándose las variables socioeconómicas que expresan la calidad de vida de las personas (educación, saneamiento, carencia de bienes y estado de la vivienda).

Los efectos marginales del modelo Probit son los que miden el cambio en la probabilidad de la variable explicada del modelo, en este caso, Pobreza. Tal como se había mencionado, los efectos estimados de las variables explicativas son iguales a los efectos esperados, con excepción de la variable Ocupado. Se evidencia que una persona que se encuentre económicamente ocupada aumenta la probabilidad de ser pobre, manteniendo todo lo demás constante. Resultado contradictorio con la realidad, debido a que se espera que, si una persona se encuentra ocupada económicamente, devengue beneficios monetarios de esa actividad. Aparte de este resultado no esperado, las variables que tuvieron mayor impacto sobre la reducción de la probabilidad que tiene un individuo de ser pobre a través de los años fueron: si la persona es casada, si donde habita se encuentran las calles asfaltadas y si posee televisión.

Dado que los efectos de las variables Edad y Edad ${ }^{2}$ son distintos, se infiere la existencia de un punto de inflexión. El punto de inflexión 
representa a partir de qué edad el efecto pasa de ser positivo a ser negativo sobre la probabilidad de ser pobre. Para el periodo 2007-2015, el punto de inflexión promedio resultó de 50 años, es decir, que de 0 a 50 años la edad posee un efecto positivo sobre la probabilidad de ser pobre y es a partir de la edad de 50 años que esta variable posee un efecto negativo sobre la probabilidad de que el individuo posea al menos el $25 \%$ de las privaciones.

En cuanto a los resultados arrojados por el modelo econométrico de Pseudo- Panel, se percibió que los hogares donde la mujer es jefa del hogar son menos propensos a ser pobres por privaciones. Este hallazgo se corrobora con el modelo de corte transversal, donde se observa que si el hombre es el jefe del hogar posee mayor probabilidad de ser pobre sobre la mujer cuando es jefa del hogar.

Se esperaba que, si la mujer fuese la jefa del hogar, la probabilidad de ser pobre fuera mayor en comparación a cuando el hombre es el jefe del hogar, debido a la segmentación existente por género del mercado laboral, específicamente en América Latina, y al compromiso doméstico que cargan sobre sus hombros las madres solteras. La obtención de un ingreso monetario resulta en promedio inferior al que puede obtenerse cuando el hombre es jefe de hogar.

A partir del pseudo-panel, se procedió a realizar un gráfico para percibir visualmente la evolución de la probabilidad de la variable definida Pobreza y, en adición, la tasa de incidencia, que mide la proporción de personas con carencias en el tiempo. Se observa que la variable Pobreza, que representa los hogares que poseen al menos el $25 \%$ de las privaciones asumidas y presentadas en el presente trabajo de investigación, posee el mismo comportamiento que la tasa de incidencia, que refleja el porcentaje de personas pobres multidimensionales.

Por otro lado, se observa un alza ligera de estas variables para el año 2008, por lo que se podría inferir que la crisis financiera de ese 
año tuvo repercusiones sobre dichas variables. Asimismo, se evidencia una caída pronunciada en el año 2010 en ambas variables. La única diferencia que presentan es que la tasa de incidencia muestra una tendencia paulatina a la baja a partir de 2012 y las variables que miden los hogares que poseen al menos el $25 \%$ de las privaciones presenta una disminución a partir de 2011. Estos resultados son consistentes con los expresados en el Primer informe de pobreza multidimensional realizado en el país, presentado a finales de junio del presente año, donde se aprecia una disminución de aproximadamente el $11 \%$ en la tasa de incidencia en el periodo 2012-2016.

\section{Bibliografía}

Alkire, S. \& Foster, J. (2007). Recuento y medición multidimensional de la pobreza . Oxford: OPHI Working Paper Series.

Banco Mundial. (2001). República Dominicana Informe sobre la pobreza: la pobreza en una economía de alto crecimiento (19862000). Santo Domingo: Banco Mundial.

Banco Mundial y Banco Interamericano de Desarrollo. (2006). Informe sobre la pobreza en la República Dominicana: logrando un crecimiento económico que beneficie a los pobres. Santo Domingo: Unidad de Gestión de Países del Caribe (BM) y Departamento Regional de Operaciones (BID).

CEPAL. (2013). La medición multidimensional de la pobreza: CEPAL y Naciones Unidas.

Comisión Económica para América Latina y el Caribe. (2008). Objetivos de desarrollo del Milenio en América Latina y el Caribe. Washington, D.C. : CEPAL.

Competitividad. (s.f.). Recuperado de http://www.competitividad. org.do/wp-content/uploads/2014/09/MPI-Final.pdf 
Deaton, A. (1995). Data and Econometric Tools For Development Analysis . Nueva Jersey: Princeton University.

Geldstein, R. (1997). Mujeres jefas del hogar. Argentina: UNICEF.

Instituto Nacional de Estadística y Censos. (2002). Medición de la pobreza método de la línea de pobreza. Costa Rica: Instituto Nacional de Estadística y Censos.

Larrañaga, O. (2007). Estudios estadísticos y prospectivos: la medición de la pobreza en dimensiones distintas al ingreso. Santiago de Chile: CEPAL.

Morillo, A. (2017). El índice de pobreza multidimensional para América Latina (IMP-LA): una aplicación para la República Dominicana 2005-2016 (informe preeliminar). Santo Domingo: Ministerio de Economía Planificación y Desarrollo.

Medina, E. (2003). Modelos de elección discreta. Madrid: Universidad Autónoma de Madrid.

Ministerio de Economía Planificación y Desarrollo. (2010). Documento base de la Propuesta de estrategia nacional de desarrollo 2010-2030: un viaje de transformación hacia un pais mejor. Santo Domingo: Ministerio de Economía Planificación y Desarrollo.

Ministerio de Economía Planificación y Desarrollo. (2010). Objetivos de desarrollo del milenio: informe seguimiento 2010. Santo Domingo: Ministerio de Economía Planificación y Desarrollo.

Ministerio de Economía, Planificación y Desarrollo y Oficina Nacional de Estadísticas. (2016). 3er Boletín de estadísticas oficiales de pobreza monetaria. Santo Domingo: Ministerio de Economía Planificación y Desarrollo. 
Morillo, A. (2008). Medición de la pobreza monetaria mediante las Encuestas de Fuerza de Trabajo (EFT) del Banco Central de la República Dominicana: Propuesta metodológica y resultados 2000-2008. Santo Domingo: Ministerio de Economía, Planificación y Desarrollo.

Morillo, A. (2012). Evolución, determinantes agregados y dimensión espacial de la pobreza monetaria en la República Dominicana, 2000-2011. Santo Domingo: Ministerio de Economía, Planificación y Desarrollo.

Morillo, A. (2014). El mapa de la pobreza en la República Dominicana 2014. Santo Domingo: Ministerio de Economía, Planificación y Desarrollo.

Morillo, A. (2015). Evolución y determinantes agregados de la pobreza monetaria en la República Dominicana, 2000-2014. Santo Domingo: Ministerio de Economía, Planificación y Desarrollo.

Programa de las Naciones Unidas para el Desarrolo (PNUD), (25 de Abril de 2016). El indice de pobreza multidimensional abre un nuevo debate que genera políticas públicas más eficientes.

Quinn, L. (2013). Determinantes de la pobreza y vulnerabilidad social en República Dominicana 2000-2012. Santo Domingo: Concurso Economía Banco Central de la República Dominicana.

Sahagún, R. (2016). Construcción y uso de un modo de pseudo-panel aplicado al análisis de la propiedad y número de autos por parte de los hogares de México. México: Instituto Nacional de Ecología y Cambio Climático.

Sen, A. (1992). Sobre conceptos y medidas de pobreza. Revista de comercio exterior, 42(4). Págs 310-322. 
Spicker, P. (1999). The international glossary on poverty. Buenos Aires: CLACSO-CROP.

SUIBEN \& Vicepresidencia de la República Dominicana.(2016). Índice de pobreza multidimensional República Dominicana. Santo Domingo: SUIBEN.

The World Bank. (2005). Introduction to poverty analysis. Washington: World Bank Institude.

Zarraga, A., Esteban, V., Orbe, S. \& Regúlez, M. (2009). Errores de especificación en la elección de los regresores. España: Departamento de Economía Aplicada III. Econometría y Estadística de la Universidad del País Vasco. 